(1)

CrossMark

\title{
Ataxia telangiectasia: why should the ERS care?
}

\author{
Jayesh M. Bhatt ${ }^{1}$, Andrew Bush ${ }^{2}$, Marjo van Gerven ${ }^{3}$, Andreea Nissenkorn $^{4}$, \\ Michael Renke ${ }^{5}$, Lian Yarlett ${ }^{6}$, Malcolm Taylor ${ }^{7}$, Thomy Tonia ${ }^{8}$, Adilia Warris ${ }^{9}$, \\ Stefan Zielen ${ }^{5}$, Shairbanu Zinna ${ }^{1}$ and Peter J.F.M. Merkus ${ }^{3}$
}

Affiliations: ${ }^{1}$ Nottingham Children's Hospital, UK Paediatric National Clinic, Nottingham University Hospitals NHS Trust, Nottingham, UK. ${ }^{2}$ Imperial College and Royal Brompton Hospital, London, UK. ${ }^{3}$ Dept of Paediatrics, Division of Respiratory Medicine, Amalia Children's Hospital, Radboud University Medical Centre, Nijmegen, The Netherlands. ${ }^{4}$ Rare Diseases Service and Pediatric Neurology Unit, Edmond and Lilly Safra Pediatric Hospital, Sheba Medical Center, Tel Ha Shomer, Sackler School of Medicine, Tel Aviv University, Tel Aviv, Israel. ${ }^{5}$ Dept of Allergology, Pneumology and Cystic Fibrosis, Children's Hospital, Goethe-University Theodor-Stern Kai, Frankfurt/Main, Germany. ${ }^{6}$ The A-T Society, IACR-Rothamsted, Harpenden, UK. ${ }^{7}$ School of Cancer Sciences, University of Birmingham, Birmingham, UK. ${ }^{8}$ Institute of Social and Preventive Medicine, University of Bern, Bern, Switzerland. ${ }^{9}$ Institute of Medical Sciences, University of Aberdeen and the Royal Aberdeen Children's Hospital, Aberdeen, UK.

Correspondence: Jayesh M. Bhatt, Nottingham Children's hospital, UK Paediatric National Clinic, Nottingham University Hospitals NHS Trust, QMC, Derby Road, Nottingham, NG7 2UH, UK. E-mail: jayeshbhattahotmail.com

0

@ERSpublications

Neurological and immunological contributions to lung disease in A-T require proactive and multidisciplinary management http://ow.ly/TqT44

Why indeed should we care? The answer is that ataxia telangiectasia $(\mathrm{A}-\mathrm{T})$ patients will present to respiratory paediatricians for diagnosis, when standard testing, if the diagnosis is not made, may do irreparable harm; and both adult and paediatric respiratory physicians will be involved in managing the respiratory disease, which is a major cause of morbidity and mortality. So there is a real "need to know" which is why the ERS convened a task force on A-T, whose findings are published in the European Respiratory Review [1].

A-T is an autosomal recessive, progressive, multisystem disease caused by mutations in the gene ATM (Ataxia-Telangiectasia Mutated) (11q22.3). This gene is expressed ubiquitously and encodes ATM kinase, a serine/threonine protein kinase, which is involved in signalling following cellular stress. It activates over a hundred proteins involved in the DNA damage response, cell cycle regulation and other pathways. ATM has important roles in neuroprotection, both adaptive and innate immunity, inflammatory responses, metabolism (e.g. insulin signalling), longevity and fertility. It has been estimated that a human cell is confronted with one million DNA lesions every day, placing DNA damage response mechanisms in a position of paramount importance. This has to be a very precise and efficient system to prevent cells with damaged DNA from dividing further or being passed on through germline mutation. The DNA double strand break (DSB) represents one of the most cytotoxic DNA lesions. DSBs can be generated by exposure to ionising radiation or various chemical compounds [2].

The Orphanet registry estimates the average prevalence of A-T to be 1 per 100000 children [3]. A-T has a large number of other complex and diverse manifestations that vary with age. Neurological symptoms, particularly progressive cerebellar ataxia and abnormalities of the eye movements appear from an early age, and malignant disease is common. Some children first come to medical attention because of recurrent sino-pulmonary infections (which affect $\sim 50 \%$ of children with A-T). Oculocutaneous telangiectasia may not occur until 4 or 5 years of age, so this clue is not available in early life. Laboratory tests may show an elevated alpha-fetoprotein level, immunological deficiencies and chromosomal instability; one lesson is to

Received: Sept 012015 | Accepted after revision: Sept 102015

Conflict of interest: None declared.

Copyright OERS 2015 
have a low threshold for the measurement of alpha-fetoprotein as a screening test, especially in young children with infections plus another sign, especially drooling or ataxia. Patients with A-T die prematurely with the leading causes of death being respiratory diseases and cancer, hence the importance of receiving respiratory management right from the start. Also, it is good to consider the diagnosis before rather than after embarking on radiological investigations such as high-resolution computed tomography (HRCT), given the sensitivity of the patients to radiation. There is no definitive treatment available at present, and no randomised controlled trials of treatment; supportive care is the mainstay of management. Median survival is 25 years [4].

A respiratory physician needs to recognise the following major lung disease phenotypes in known A-T patients.

\section{Immune dysfunction leading to recurrent upper and lower respiratory infections}

Immune dysfunction in A-T leads to recurrent upper and lower respiratory infections, which in turn can lead to bronchiectasis. The immune deficiency in A-T is highly variable and affects both B- and T-cells. Radiologically confirmed bronchiectasis seems to be established by the end of first decade [5-7]. The prevalence of bronchiectasis varies from $10 \%$ [5] to $47 \%$ [6]. The disadvantage of computed tomography is radiation exposure, and it is important that the pulmonologist thinks critically about whether HRCT will truly change management, and is thus essential, or whether clinical management (airway clearance, antibiotics) is really influenced by this investigation in a child with a chronic wet cough. Magnetic resonance imaging may be valuable in the assessment of the extent and severity of pulmonary changes in children and adults with A-T [8], and is likely to become the modality of choice in the future. Experience suggests that proactive monitoring and aggressive multidisciplinary treatment delays the onset of or stabilises bronchiectasis. The microbiology of the respiratory tract in patients with $\mathrm{A}-\mathrm{T}$ is unlike that of other primary immune deficiencies and has more similarities to that seen in patients with cystic fibrosis (CF) $[5,9]$. Other similarities to CF are that the systemic spread of infectious organisms is rare, and opportunistic infections such as Pneumocystis jiroveci are not observed, for reasons that are unclear $[5,6]$.

There is a large variation in the practice of prescribing prophylactic antibiotics for patients with A-T and this is considered on an individualised basis when the burden of respiratory infections is high. However, microbiological resistance and side-effects should be always borne in mind.

The decision to commence immunoglobulin replacement therapy is taken on clinical grounds in those children who have a history of recurrent respiratory infections and low specific antibody responses despite booster immunisations [10]. Routine childhood immunisations, including attenuated live viral vaccines, will usually have been given prior to the diagnosis of A-T and/or recognition of the associated immune deficiency [11]. Reports of adverse events in patients with A-T after the administration of live vaccines are rare and, for the most part, vaccines are well-tolerated [5].

\section{Lung disease associated with dysfunctional swallow and inefficient cough}

Lung disease associated with dysfunctional swallow and inefficient cough due to neurodegenerative deficits increases the risk for aspiration, and consequently may cause or worsen bronchiectasis. In A-T, dysphagic problems commonly emerge in the second decade of life [12]. These are multifactorial: ataxia, hyperkinesis, and bulbar dysfunction due to cerebellar involvement all lead to dys-coordination of the oral-motor and swallowing movements. The onset of dysphagia appears to coincide with a decrease in nutritional status, although it was not possible to distinguish between nutritional deficiency as a cause or effect of the dysphagia in a cross sectional study [13]. Patients with A-T exhibit high rates of malnutrition, a short stature and reduced lean body mass [14-17]. The numerous factors that, together, contribute to poor growth include: neurodegeneration; limited food intake with progressive disease; dysphagia and/or swallowing incoordination; limited physical activity; hormonal changes such as hypogonadism, insulin resistance, glucose intolerance, abnormal expression of IGF1 (somatomedin C), and low levels of IGFBP3 (insulin-like growth factor binding protein-3); infections and an associated hyper-catabolic state [15, 18]. Regular reviews by a speech and language therapist are vital for clinical assessment and appropriate early advice for changes in feeding routine and about body posture can be given. Radiographic confirmation of swallowing dysfunction can be obtained by judicious use of a video fluoroscopic swallow study protocol that has been developed to minimise radiation exposure [13]. Ongoing concerns regarding aspiration and/ or adequacy of nutrition should prompt referral for gastrostomy insertion. While it is difficult to define the optimal age for gastrostomy placement, significant progressive decline in weight, length and body mass index (BMI) z-scores appears to start from the of age 8 years (E. Stewart, et al., Nottingham Children's Hospital, UK Paediatric National Clinic, Nottingham University Hospitals, NHS Trust, Nottingham, UK; personal communication). Even though a significant increase in weight or BMI z-score may not be seen post-gastrostomy insertion in $\mathrm{A}-\mathrm{T}$, an almost universal improvement in health-related quality of life scores 
is reported [19]. In addition, delayed insertion of a gastrostomy is more likely to be associated with worse outcomes [19].

\section{Interstitial lung disease/pulmonary fibrosis}

The aetiology and pathogenesis of interstitial lung disease (ILD) and pulmonary fibrosis in A-T patients is unknown; however, it is distinctive in its histology and bronchoalveolar lavage findings. It needs to be considered in any patient with A-T who has chronic respiratory symptoms, especially a persistent dry cough, as only treatment with systemic corticosteroids early in the course of the illness is associated with clinical and radiographic improvement [20]. Other non-infective causes of diffuse lung diseases (which may mimic ILD) in A-T include pulmonary parenchymal involvement secondary to lymphoma [21, 22] and pulmonary fibrosis secondary to chemotherapy for the treatment of a malignancy [23].

\section{Respiratory monitoring}

The underlying neurological involvement [24] can make it difficult to obtain reproducible, reliable dynamic lung function tests that require any effort and coordination. However, pulmonary function testing with certain modifications can be carried out reliably and reproducibly by many patients with A-T, which may be useful in tracking the rate of decline in lung function over time [6, 24-27]. Incoordination and fatigue are characteristic of A-T and contribute to inefficient cough and aspiration, both of which are significant contributors to lung disease. Mucociliary clearance is impaired following recurrent infections and aspiration. Measures to improve impaired mucociliary clearance and cough clearance, and to prevent atelectasis are likely to be beneficial in A-T patients, especially those with more advanced neurological decline. Physiotherapy advice regarding which techniques to adopt to optimise airway clearance before irreversible structural lung damage occurs is desirable, although there is no firm evidence base to give specific advice for A-T. Some form of regular exercises, even in younger children who are still mobile and not yet on a downward trajectory of pulmonary and neurological decline, are usually advised to parents and carers by most experts who look after children with A-T. Inspiratory muscle training [28] and cough assist devices (I. Sarouk, The Edmond and Lily Safra Children's Hospital, Sheba Medical Center, RamatGan, Israel; personal communication) have anecdotally been used with some success in patients with A-T.

\section{Other contributors to pulmonary morbidity}

Scoliosis is present in just over 20\% of classical A-T patients [29] and may require spinal surgery. Maintaining assisted ambulation as long as possible might prevent scoliosis and further deterioration of lung function, but further studies are needed to sustain this clinical observation.

Patients with A-T may require general anaesthesia for, for example, a bronchoscopy, lung biopsy, gastrostomy insertion or scoliosis surgery. There is scant evidence, to date, on the anaesthetic risks in A-T and a full evaluation prior to anaesthesia should be carried out to stabilise and optimise the patients [30].

\section{Organisation of care for lung disease in A-T}

Respiratory disease in $\mathrm{A}-\mathrm{T}$ is complex and its understanding and management requires multidisciplinary input. Current approaches are based on minimal best available evidence in A-T, and extrapolation from other more common disorders such as CF and antibody deficiencies. The combined neurological and immunological contributions to lung disease in A-T, however, are unlike that in $\mathrm{CF}$ and emphasise the need for multidisciplinary expertise to manage this devastating disease. Specialised national clinics provide multidisciplinary expertise to manage patients with A-T and this has been synthesised in this task force report [1]. But above all, think of the possibility of an early diagnosis, before multiple radiological investigations have been performed!

\section{References}

1 Bhatt JM, Bush A, van Gerven M, et al. A statement on the multidisciplinary respiratory management of ataxia telangiectasia. Eur Respir Rev 2015; 24: 565-581.

2 Chaudhary MW, Al-Baradie RS. Ataxia-telangiectasia: future prospects. Appl Clin Genet 2014; 7: 159-167.

3 Orphanet. Ataxia-telangiectasia. www.orpha.net/consor/cgi-bin/OC_Exp.php?lng=EN\&Expert=100 Date last accessed: March, 2015. Date last updated: October 14, 2015.

4 Crawford TO. Survival probability in ataxia telangiectasia. Arch Dis Child 2005; 91: 610-611.

5 Schroeder SA, Zielen S. Infections of the respiratory system in patients with ataxia-telangiectasia. Pediatr Pulmonol 2014; 49: 389-399.

6 Bott L, Lebreton J, Thumerelle C, et al. Lung disease in ataxia-telangiectasia. Acta Paediatr 2007; 96: 1021-1024.

7 Micol R, Ben Slama L, Suarez F, et al. Morbidity and mortality from ataxia-telangiectasia are associated with ATM genotype. J Allergy Clin Immunol 2011; 128: 382-389.

8 Montella S, Mollica C, Finocchi A, et al. Non invasive assessment of lung disease in ataxia telangiectasia by high-field magnetic resonance imaging. J Clin Immunol 2013; 33: 1185-1191.

9 Bhatt JM, Bush A. Microbiological surveillance in lung disease in ataxia telangiectasia. Eur Respir J 2014; 43: $1797-1801$. 
Davies EG. Update on the management of the immunodeficiency in ataxia-telangiectasia. Expert Rev Clin Immunol 2009; 5: 565-575.

11 Nowak-Wegrzyn A, Crawford TO, Winkelstein JA, et al. Immunodeficiency and infections in ataxia-telangiectasia. J Pediatr 2004; 144: 505-511.

12 Crawford TO, Mandir AS, Lefton-Greif MA, et al. Quantitative neurologic assessment of ataxia-telangiectasia. Neurology 2000; 54: 1505-1509.

13 Lefton-Greif MA, Crawford TO, Winkelstein JA, et al. Oropharyngeal dysphagia and aspiration in patients with ataxia-telangiectasia. J Pediatr 2000; 136: 225-231.

14 Schubert R, Reichenbach J, Zielen S. Growth factor deficiency in patients with ataxia telangiectasia. Clin Exp Immunol 2005; 140: 517-519.

15 Voss S, Pietzner J, Hoche F, et al. Growth retardation and growth hormone deficiency in patients with Ataxia telangiectasia. Growth Factors 2014; 32: 123-129.

16 da Silva R, dos Santos-Valente EC, Burim Scomparini F, et al. The relationship between nutritional status, vitamin $\mathrm{A}$ and zinc levels and oxidative stress in patients with ataxia-telangiectasia. Allergol Immunopathol (Madr) 2014; 42: 329-335.

17 Ross LJ, Capra S, Baguley B, et al. Nutritional status of patients with ataxia-telangiectasia: a case for early and ongoing nutrition support and intervention. J Paediatr Child Health 2015; 51: 802-807.

18 Peretz S, Jensen R, Baserga R, et al. ATM-dependent expression of the insulin-like growth factor-I receptor in a pathway regulating radiation response. Proc Natl Acad Sci USA 2001; 98: 1676-1681.

19 Lefton-Greif MA, Crawford TO, McGrath-Morrow S, et al. Safety and caregiver satisfaction with gastrostomy in patients with Ataxia Telangiectasia. Orphanet J Rare Dis 2011; 6: 23

20 Schroeder SA, Swift M, Sandoval C, et al. Interstitial lung disease in patients with ataxia-telangiectasia. Pediatr Pulmonol 2005; 39: 537-543.

21 Canny GJ, Roifman C, Weitzman S, et al. A pulmonary infiltrate in a child with ataxia telangiectasia. Ann Allergy 1988; 61: 422-423.

22 Yalçin B, Kutluk MT, Sanal O, et al. Hodgkin's disease and ataxia telangiectasia with pulmonary cavities. Pediatr Pulmonol 2002; 33: 399-403.

23 Chen RL, Wang PJ, Hsu YH, et al. Severe lung fibrosis after chemotherapy in a child with ataxia-telangiectasia. J Pediatr Hematol Oncol 2002; 24: 77-79.

24 McGrath-Morrow S, Lefton-Greif M, Rosquist K, et al. Pulmonary function in adolescents with ataxia telangiectasia. Pediatr Pulmonol 2008; 43: 59-66.

25 McGrath-Morrow SA, Lederman HM, Aherrera AD, et al. Pulmonary function in children and young adults with ataxia telangiectasia. Pediatr Pulmonol 2014; 49: 84-90.

26 Berkun Y, Vilozni D, Levi Y, et al. Reversible airway obstruction in children with ataxia telangiectasia. Pediatr Pulmonol 2010; 45: 230-235.

27 Vilozni D, Berkun Y, Levi Y, et al. The feasibility and validity of forced spirometry in ataxia telangiectasia. Pediatr Pulmonol 2010; 45: 1030-1036.

28 Félix E, Gimenes AC, Costa-Carvalho BT. Effects of inspiratory muscle training on lung volumes, respiratory muscle strength, and quality of life in patients with ataxia telangiectasia. Pediatr Pulmonol 2014; 49: 238-244.

29 Ataxia-Telangiectasia Society. Ataxia-telangiectasia in children: guidance on diagnosis and clinical care. www.atsociety. org.uk/data/files/William/A-T_Clinical_Guidance_Document_Final.pdf Date last accessed: March 17, 2015. Date last updated: October, 2014

30 Lockman JL, Iskander AJ, Bembea M, et al. Anesthetic and perioperative risk in the patient with ataxiatelangiectasia. Paediatr Anaesth 2012; 22: 256-262. 